\title{
Linear Response to Perturbation of Nonexponential Renewal Processes
}

\author{
Francesco Barbi, ${ }^{1}$ Mauro Bologna, ${ }^{2}$ and Paolo Grigolini ${ }^{1,3,4}$ \\ ${ }^{1}$ Dipartimento di Fisica "E. Fermi," Universitá di Pisa, Largo Pontecorvo, 356127 Pisa, Italy \\ ${ }^{2}$ Departamento de Física, Universidad de Tarapacá, Campus Velásquez, Velásquez 1775, Casilla 7-D, Arica, Chile \\ ${ }^{3}$ Center for Nonlinear Science, University of North Texas, P.O. Box 311427, Denton, Texas 76203-1427, USA \\ ${ }^{4}$ Istituto dei Processi Chimico Fisici del CNR, Area della Ricerca di Pisa, Via G. Moruzzi, 56124 Pisa, Italy
} (Received 12 July 2005; published 23 November 2005)

\begin{abstract}
We study the linear response of a two-state stochastic process, obeying the renewal condition, by means of a stochastic rate equation equivalent to a master equation with infinite memory. We show that the condition of perennial aging makes the response to coherent perturbation vanish in the long-time limit.
\end{abstract}

DOI: 10.1103/PhysRevLett.95.220601

PACS numbers: 05.40.Fb, 02.50.-r, 82.20.Uv

Many complex processes generate erratic jumps back and forth from a state "on" to a state "off." We limit ourselves to quoting ionic channel fluctuations [1-3], currently triggering the search for a form of stochastic resonance valid also in the nonexponential case [4], and the intermittency of blinking nanocrystals [5,6]: It has been assessed that the intermittent fluorescence of these materials obeys the renewal theory [7]; namely, a jump from one to the other state has the effect of resetting the system's memory to zero. The nonexponential nature of the distribution of sojourn times makes this renewal process nonergodic and generates aging effects that are the object of an increasing theoretical interest $[8,9]$. Similar properties are found with surface-enhanced Raman spectra of single molecules [10].

The authors of [11-13] studied the joint effect of aging and perturbation on a process of subdiffusion, without establishing, however, a direct connection with the issue of non-Poisson stochastic resonance [4]. Here we fill this gap by means of a stochastic master equation, with no time convolution. This method might be extended to an arbitrarily large number of states and to the case of arbitrarily intense perturbation.

Let us assume that the distributions of on and off times are identical. We assign to the survival probability (SP) of this process, $\Psi(t)$, the inverse power law form

$$
\Psi(t)=\left(\frac{T}{T+t}\right)^{\mu-1},
$$

with $\mu>1$. This corresponds to the joint action of the time-dependent rate $[14,15] q(t)=q_{0} /\left(1+q_{1} t\right)$, with $q_{0}=(\mu-1) / T$ and $q_{1}=1 / T$, and of a resetting prescription. To illustrate this condition, let us imagine the random drawing of a number from the interval $I=[0,1]$ at discrete times $i=1,2, \ldots$ The interval $I$ is divided into two parts, $I_{1}$ and $I_{2}$, with $I_{1}$ ranging from 0 to $p_{i}$, and $I_{2}$ ranging from $p_{i}$ to 1 . Note that $p_{i}=1-q_{i}<1$ and $q_{i} \ll$ 1 , and, as a consequence, the number of times we keep drawing numbers from $I_{1}$, without moving to $I_{2}$, is very large.
Let us evaluate the distribution of these persistence times, and let us discuss under which conditions we get the SP of Eq. (1). The SP function is the probability of remaining in $I_{1}$ after $n$ drawings and is consequently given by

$$
\Psi(n)=\prod_{i=1}^{n} p_{i}
$$

Using the condition $q_{i} \ll 1$, and evaluating the logarithm of both terms of Eq. (2), we obtain

$$
\log (\Psi(n))=-\sum_{i=1}^{n} q_{i}
$$

The condition $q_{i} \ll 1$ implies that $i$ and $n$ of Eq. (3) are so large as to make $q_{i}$ virtually identical to a function of the continuous time $t, q_{i}=q(t)=q_{0} / r\left[1+q_{1} \Delta \tau(t)\right]$, with $\Delta \tau(t)=t$. Thus, Eq. (3) yields the SP of Eq. (1), and the corresponding waiting time distribution density, $\psi(\tau)$, reads

$$
\psi(\tau)=(\mu-1) \frac{T^{\mu-1}}{(\tau+T)^{\mu}} .
$$

We denote as collisions the rare drawings of a number from $I_{2}$, followed by resetting. Thus the collisions occurring at times $\tau_{1}, \tau_{1}+\tau_{2}, \ldots$, yield $\Delta \tau(t)=t, 0<t<\tau_{1}$, $\Delta \tau(t)=\left(t-\tau_{1}\right), \tau_{1}<t<\tau_{1}+\tau_{2}$, and so on. Note that $\Delta \tau(0)=0$ means that we prepare the system at time $t=0$. We adopt a coin tossing prescription to decide whether to keep or to change sign, after any collision.

The state of the system after the $n$th collision is described by the two-dimensional vector $\mathbf{P}(n)$, whose components, $P_{1}$ and $P_{2}$, are the probabilities of finding the system in the corresponding states. The $n$th collision produces the change

$$
\mathbf{P}(n)=\mathbf{M P}(n-1),
$$

where 


$$
\mathbf{M}=\left(\begin{array}{ll}
1 / 2 & 1 / 2 \\
1 / 2 & 1 / 2
\end{array}\right)
$$

Equation (5) refers to an integer-time representation. We convert this collisional process into a virtually continuoustime representation by relating the collisions to the absolute time at which they occur, as done in [16]. We obtain

$$
\frac{d}{d t} \mathbf{P}(t)=-\int_{0}^{t} d \tau \Phi(t-\tau) \mathbf{K} \mathbf{P}(\tau) .
$$

The matrix $\mathbf{K}$ is defined by

$$
\mathbf{K} \equiv \frac{1}{2}\left(\begin{array}{cc}
1 & -1 \\
-1 & 1
\end{array}\right)
$$

and the memory kernel $\Phi(t)$ is related to $\psi(t)$ in the Laplace domain through

$$
\hat{\Phi}(u)=\frac{u \hat{\psi}(u)}{1-\hat{\psi}(u)}
$$

where $\hat{\Phi}(u)$ and $\hat{\psi}(u)$ denote the Laplace transforms of $\Phi(t)$ and $\psi(t)$, respectively.

Now, let us define the quantity

$$
\Pi(t)=P_{1}(t)-P_{2}(t)=2 P_{1}(t)-1 .
$$

Using Eq. (7), we get for the Laplace transform of $\Pi(t)$, $\hat{\Pi}(u)$, the following expression:

$$
\hat{\Pi}(u)=\frac{1}{u+\hat{\Phi}(u)},
$$

which, in turn, using Eq. (9), gives $\hat{\Pi}(u)=\hat{\Psi}(u)$, namely, the key property

$$
\Pi(t)=\Psi(t) .
$$

There exists another way to move from the discrete time $n$ to time $t$. This is through the following Markov but stochastic master equation:

$$
\begin{aligned}
& \sigma_{1}(t+1)-\sigma_{1}(t)=-\frac{r_{+}(t)}{2} \sigma_{1}(t)+\frac{r_{-}(t)}{2} \sigma_{2}(t), \\
& \sigma_{2}(t+1)-\sigma_{2}(t)=-\frac{r_{-}(t)}{2} \sigma_{2}(t)+\frac{r_{+}(t)}{2} \sigma_{1}(t) .
\end{aligned}
$$

Let us study first this equation when no perturbation occurs. In this case, we set $r_{+}(t)=r_{-}(t)=r(t)$, with $r(t)$ being a function of time always vanishing except when a collision occurs, where it then gets the value of 1 . We adopt the notation

$$
\exp \left(-\int_{t^{\prime}}^{t} d t^{\prime \prime} r\left(t^{\prime \prime}\right)\right) \equiv \exp \left(t, t^{\prime}\right) \equiv \prod_{i=0}^{t-t^{\prime}}[1-r(t-i)] .
$$

Note that with these definitions we obtain

$$
\begin{aligned}
\frac{d}{d t^{\prime}} \exp \left(t, t^{\prime}\right) & =\exp \left(t, t^{\prime}+1\right)-\exp \left(t, t^{\prime}\right) \\
& =-r\left(t^{\prime}+1\right) \exp \left(t, t^{\prime}\right)
\end{aligned}
$$

which for very large values of $t^{\prime}$ is equivalent to

$$
\frac{d}{d t^{\prime}} \exp \left(-\int_{t^{\prime}}^{t} r\left(t^{\prime \prime}\right) d t^{\prime \prime}\right)=-\exp \left(-\int_{t^{\prime}}^{t} r\left(t^{\prime \prime}\right) d t^{\prime \prime}\right) r\left(t^{\prime}\right),
$$

namely, the ordinary continuous-time prescription.

To confirm the equivalence of Eq. (13) with Eq. (7) let us prove that the statistical average of $\Sigma(t) \equiv \sigma_{1}(t)-$ $\sigma_{2}(t)=2 \sigma_{1}(t)-1$ yields $\Pi(t)$ of Eq. (10). Let us assume that all the systems are located at the beginning of their sojourn in either the state on or the state off, so as to fit the prescription of preparing the system at $t=0$. Thanks to Eq. (13), we obtain

$$
\Sigma(t)=\exp \left(-\int_{0}^{t} r\left(t^{\prime}\right) d t^{\prime}\right)
$$

which yields

$$
\langle\Sigma(t)\rangle=\Psi(t) .
$$

In fact, $\Sigma(t)$, due to Eq. (14), drops abruptly from 1 to 0 when a collision occurs. Thus, its mean value becomes identical to the survival probability $\Psi(t)$. Using Eq. (12), we conclude that $\langle\Sigma(t)\rangle=\Pi(t)$.

Although the approach of this Letter is not confined to the linear response, let us study the effect of a weak perturbation on $T$, by making both $q_{0}$ and $q_{1}$ depend on time so as to keep $\mu$ fixed. As in Ref. [11], we set a bias at the moment of a collision, without perturbing the temporal sequence $\tau_{1}, \tau_{2}, \ldots$. This linear response assumption is realized by expressing Eq. (13) in terms of $r_{ \pm}(t)=r(t) \times$ $\left[1+\epsilon F_{ \pm}(t)\right]$, where $\epsilon$ is the perturbation strength and the functions $F_{ \pm}(t)$ describe the perturbation action on the corresponding states. Using Eq. (13) we obtain

$$
\frac{d}{d t} \Sigma(t)=-r(t)[1+\epsilon S(t)] \Sigma(t)-r(t) \epsilon f(t),
$$

where $S(t) \equiv\left[F_{+}(t)+F_{-}(t)\right] / 2$ and $f(t) \equiv\left[F_{+}(t)-\right.$ $\left.F_{-}(t)\right] / 2$. We assume that the perturbation strength $\epsilon$ is so small as to replace $1+\epsilon S(t)$ with 1 . Solving the resulting approximated form of Eq. (19), we arrive at

$$
\begin{aligned}
\Sigma(t)= & -\epsilon \int_{0}^{t} d t^{\prime} \exp \left(-\int_{t^{\prime}}^{t} r\left(t^{\prime \prime}\right) d t^{\prime \prime}\right) r\left(t^{\prime}\right) f\left(t^{\prime}\right) \\
& \left.+\Sigma(0) \exp \left(-\int_{0}^{t} r\left(t^{\prime}\right) d t^{\prime}\right)\right)
\end{aligned}
$$

with the fluctuation of $r(t)$ being the same as in the absence of perturbation.

We are interested in the mean value of $\Sigma(t)$, namely, $\Pi(t)$. This leads us to write 


$$
\Pi(t)=-\epsilon \int_{0}^{t} \chi\left(t, t^{\prime}\right) f\left(t^{\prime}\right) d t^{\prime}+\Pi(0) \Psi(t),
$$

where

$$
\chi\left(t, t^{\prime}\right)=\frac{d}{d t^{\prime}} \Psi\left(t, t^{\prime}\right),
$$

with $\Psi\left(t, t^{\prime}\right)$ being the characteristic function

$$
\Psi\left(t, t^{\prime}\right) \equiv\left\langle\exp \left(-\int_{t^{\prime}}^{t} d t^{\prime \prime} r\left(t^{\prime \prime}\right)\right)\right\rangle
$$

With $\Pi(0)=0$, Eq. (21) becomes formally identical to the common linear response prescription [17], with, however, aging violating the stationary condition $\chi\left(t, t^{\prime}\right)=$ $\chi\left(t-t^{\prime}\right)$.

Using the same arguments as those earlier adopted to prove Eq. (18), we show that $\Psi\left(t, t^{\prime}\right)$ coincides with the survival probability of age $t^{\prime}$ of Ref. [18]. According to [18], the function $\Psi\left(t, t^{\prime}\right)$ coincides with the aging correlation function of the dichotomous signal under study, $C\left(t, t^{\prime}\right)$. By means of the stationary assumption, we recover the results of Refs. $[4,19,20]$ and we realize why these earlier results go beyond the Green-Kubo prediction $[21,22]$.

In the case $\mu<2$, the stationary condition cannot be realized, not even in principle, while in the case $\mu>2$, it is possible, even if $\mu<3$ makes the relaxation to equilibrium very slow [23]. Let us study first the more traditional condition $\mu>2$, and let us switch perturbation on after the transient process necessary to reach equilibrium. We have [24]

$$
\chi\left(t, t^{\prime}\right)=-\psi_{\infty}\left(t-t^{\prime}\right)=-(\mu-2) \frac{T^{\mu-2}}{\left(t-t^{\prime}+T\right)^{\mu-1}} .
$$

In this case, the stationary correlation function of the on and off fluctuation, $C\left(t-t^{\prime}\right)$, exists and has the analytical form $[T /(t+T)]^{\mu-2}$, thereby yielding

$$
\psi_{\infty}\left(t-t^{\prime}\right)=-\frac{d}{d t} C\left(t-t^{\prime}\right) .
$$

Note that the linear response of Eq. (21), with $-\chi\left(t, t^{\prime}\right)$ replaced by $\psi_{\infty}\left(t-t^{\prime}\right)$ of Eq. (25), coincides with the prescription of the phenomenological approach of Ref. [4]. In the case where $f(t)=\Theta(t)$, with $\Theta(t)$ denoting the unit step function, by plugging Eq. (25) into Eq. (21), we obtain

$$
\Pi(\infty)=\epsilon,
$$

and consequently the constant drift of Ref. [19] (the subdiffusional map of Ref. [20], in this case, yields the same result).

In the case where $f(t)=\Theta(t) \cos (\omega t)$, namely, the case of stochastic resonance discussed by the authors of Ref. [4], by using the expression for the Laplace transform of $\psi_{\infty}(t)$ and the convolution theorem, we obtain for the Laplace transform of $\Pi(t)$ the following result:

$$
\hat{\Pi}(u)=\epsilon\left(1+c T^{\mu-2} u^{\mu-2}\right) \frac{u}{\left(u^{2}+\omega^{2}\right)},
$$

where $c \equiv-\Gamma(3-\mu)$. Using the fractional derivative method of Ref. [25], we evaluate the anti-Laplace transform of $\hat{\Pi}(u)$ of Eq. (27). In the case $\omega T \ll 1$, we obtain $[26,27]$

$$
\begin{aligned}
\Pi(t) \approx & \epsilon\left[1+(\omega T)^{\mu-2} \Gamma(3-\mu) \cos \frac{\pi}{2} \mu\right] \cos \omega t \\
& -\epsilon(\omega T)^{\mu-2} \Gamma(3-\mu) \sin \frac{\pi}{2} \mu \sin \omega t .
\end{aligned}
$$

As pointed out earlier, the theory of this Letter also applies to the aging case and to the condition $\mu<2$, which makes aging a perennial condition of renewal systems. In this case, using dynamical arguments [26] we identify the susceptibility of Eq. (21) with the aged $\psi\left(t, t^{\prime}\right)$. It is known $[18,28]$ that the exact expression for $\psi\left(t, t^{\prime}\right)$ is

$$
\psi\left(t, t^{\prime}\right)=\psi(t)+\sum_{n=1}^{\infty} \int_{0}^{t^{\prime}} \psi_{n}(\tau) \psi(t-\tau) d \tau,
$$

where $\psi_{n}(t)$ denotes the probability density of occurrence, at time $t$, of the last of a sequence of $n$ collisions. Let us go back to Eq. (21) and let us set $\Pi(0)=0$. This has the effect of producing a condition where a nonvanishing $\Pi(t)$ appears only as a perturbation effect. For the Laplace transform of $\Pi(t)$, we obtain the following expression:

$$
\hat{\Pi}(u)=-\epsilon \operatorname{Re}(\hat{E}(u)),
$$

where $\hat{E}(u)$ is the Laplace transform of

$$
E(t) \equiv \int_{0}^{t} d t^{\prime} \psi\left(t, t^{\prime}\right) \exp \left(-i \omega t^{\prime}\right)
$$

After some algebra, we find

$$
\hat{E}(u)=\frac{i}{\omega} \frac{[\hat{\psi}(u+i \omega)-\hat{\psi}(u)]}{[1-\hat{\psi}(u+i \omega)]} .
$$

It is of some interest to study Eq. (32), with $\mu>2$ and $\omega T \ll 1$. Using the imaginary Laplace transforms of $\psi(t)$ [29], for $u \rightarrow 0$ we get an analytical form for $\hat{E}(u)$; with this analytical form plugged into Eq. (30), we recover Eq. (28) in the same limiting condition, namely, the Laplace transform of $\epsilon \cos (\omega t)$. This means that perturbing the system in the infinitely aged condition, or keeping it under the perturbation influence, from the preparation to the infinitely aged condition, yields the same result.

It is interesting to remark that Eq. (32) establishes the effect of the step function perturbation in the case $\mu<2$. As a first step, we write Eq. (32) in the limiting condition $\omega \rightarrow 0$. This first step yields $\hat{E}(u)=-[d \hat{\psi}(u) / d u] /$ $[1-\hat{\psi}(u)]$. As a second step, we set $u \rightarrow 0$. By using the expression of $\hat{\psi}(u)$, in this limiting condition, with $\mu<2$, 
and by means of Eq. (30), we obtain the final result $\hat{\Pi}(u)=$ $-\epsilon\left[(\mu-1) / u+c / u^{\mu-1}\right]$, in the Laplace domain, and $\Pi(t) \approx-\epsilon\left[(\mu-1)+k / t^{2-\mu}\right]$, in time domain (the symbols $c$ and $k$ denote constants).

To study the effect of $f(t)=\Theta(t) \cos (\omega t)$, let us make the assumption $\omega T \ll 1$, which, in the case of $\mu>2$, turns $\hat{\Pi}(u)$ into the Laplace transform of $\epsilon \cos (\omega t)$, as shown by Eq. (28). In the case $\mu<2$, the assumption $\omega T \ll 1$ allows us to obtain

$$
\hat{\Pi}(u)=\epsilon \operatorname{Re}\left\{\frac{i}{\omega}\left[1-\left(\frac{u}{u+i \omega}\right)^{\mu-1}\right]\right\} .
$$

Using the fractional derivative method of Ref. [25], we find [26]

$$
\begin{aligned}
\Pi(t) & =-\epsilon \operatorname{Re}\left[\sum_{n=1}^{\infty}\left(\begin{array}{c}
\mu-1 \\
n
\end{array}\right) \frac{(-i \omega t)^{n-1}}{(n-1) !} e^{-i \omega t}\right] \\
& =-\epsilon \operatorname{Re}\left[(\mu-1) F(2-\mu, 2, i \omega t) e^{-i \omega t}\right],
\end{aligned}
$$

where $F(\alpha, \beta, z)$ is the confluent hypergeometric function [30]. In the special case $\mu=3 / 2$, of interest for the physics of blinking quantum dots $[5,6]$, the method of Ref. [25] yields [26]

$$
\Pi(t)=-\frac{\epsilon}{2}\left[J_{0}\left(\frac{\omega t}{2}\right) \cos \left(\frac{\omega t}{2}\right)-J_{1}\left(\frac{\omega t}{2}\right) \sin \left(\frac{\omega t}{2}\right)\right],
$$

with $J_{n}(\cdots)$ denoting the Bessel function, and, in general,

$$
\Pi(t) \approx \epsilon \frac{\cos \left(\frac{\pi}{2} \mu+\omega t\right)}{\Gamma(\mu-1)(\omega t)^{2-\mu}},
$$

which means that the condition $\mu<2$ annihilates the response coherence in the long-time limit.

In conclusion, the approach to the response of nonexponential renewal processes to external perturbation illustrated in this Letter meets the request for extending the theory of stochastic resonance to the non-Poisson case, with no stationary constraint [4]. This approach shows that the stochastic resonance dies out, if the perennial aging condition applies. We afford also the attractive possibility of moving beyond the linear response.

P. G. acknowledges Welch for financial support through Grant No. 70525.

[1] W. Nadler and D. L. Stein, Proc. Natl. Acad. Sci. U.S.A. 88, 6750 (1991).

[2] I. Goychuck and P. Hänggi, Proc. Natl. Acad. Sci. U.S.A. 99, 3552 (2002).

[3] A. Fuliński, Z. Grzywna, Z. Siwy, and P. N. R. Usherwood, Phys. Rev. E 58, 919 (1998).
[4] I. Goychuk and P. Hänggi, Phys. Rev. Lett. 91, 070601 (2003).

[5] M. Kuno, D. P. Fromm, H. F. Hamann, A. Gallagher, and D. J. Nesbitt, J. Chem. Phys. 112, 3117 (2000).

[6] K. T. Shimizu, R. G. Neuhaser, C. A. Leatherdale, S. A. Empedocles, W. K. Woo, and M.G. Bawendi, Phys. Rev. B 63, 205316 (2001).

[7] X. Brokmann, J. P. Hermier, G. Messin, P. Desbiolles, J.-P. Bouchaud, and M. Dahan, Phys. Rev. Lett. 90, 120601 (2003).

[8] G. Margolin and E. Barkai, Phys. Rev. Lett. 94, 080601 (2005).

[9] E. Lutz, Phys. Rev. Lett. 93, 190602 (2004).

[10] A. R. Bizzarri and S. Cannistraro, Phys. Rev. Lett. 94, 068303 (2005).

[11] I. M. Sokolov, A. Blumen, and J. Klafter, Europhys. Lett. 56, 175 (2001).

[12] I. M. Sokolov, A. Blumen, and J. Klafter, Physica (Amsterdam) 302A, 268 (2001).

[13] E. Barkai and Y.-C. Cheng, J. Chem. Phys. 118, 6167 (2003).

[14] I. Goychuk, Phys. Rev. E 70, 016109 (2004).

[15] D. R. Cox, Renewal Theory (Methuen, London, 1962).

[16] P. Allegrini, G. Aquino, P. Grigolini, L. Palatella, and A. Rosa, Phys. Rev. E 68, 056123 (2003).

[17] P. Hänggi and H. Thomas, Phys. Rep. 88, 207 (1982).

[18] P. Allegrini, G. Aquino, P. Grigolini, L. Palatella, A. Rosa, and B. J. West, Phys. Rev. E 71, 066109 (2005).

[19] G. Trefán, E. Floriani, B. J. West, and P. Grigolini, Phys. Rev. E 50, 2564 (1994).

[20] E. Barkai and J. Klafter, Phys. Rev. Lett. 79, 2245 (1997).

[21] E. Barkai and V. N. Fleurov, Phys. Rev. E 58, 1296 (1998).

[22] We note that to recover the Green-Kubo prediction, $\chi\left(t, t^{\prime}\right)=\chi\left(t-t^{\prime}\right) \propto C\left(t-t^{\prime}\right)$, we should have made the assumption $r_{ \pm}(t)=r(t) \pm \epsilon f(t)$, which does not properly reflect the physics of these processes.

[23] P. Allegrini, J. Bellazzini, G. Bramanti, M. Ignaccolo, P. Grigolini, and J. Yang, Phys. Rev. E 66, 015101 (2002).

[24] G. Aquino, M. Bologna, P. Grigolini, and B. J. West, Phys. Rev. E 70, 036105 (2004).

[25] B. J. West, M. Bologna, and P. Grigolini, Physics of Fractal Operators (Springer-Verlag, New York, 2003).

[26] Details on these calculations will be given in a more extended publication.

[27] With $\omega T \gg 1$ we obtain $\Pi(t) \approx \epsilon(\mu-2) \sin (\omega t) /(\omega T)$, in qualitative agreement with the resonantlike effect of Ref. [4], which is expected become quantitative when the condition $\epsilon \propto \log (1 / T)$ is taken into account.

[28] C. Godréche and J. Luck, J. Stat. Phys. 104, 489 (2001).

[29] J. Klafter and G. Zumofen, Physica (Amsterdam) 196A, 102 (1993).

[30] M. Abramowitz and I. A. Stegun, Handbook of Mathematical Functions (Dover, New York, 1972). 